\title{
Ulnar nerve tumours of the hand in childhood
}

\author{
N.P.C. C A V A N A G H A N D J.R. P I N C O T T \\ From The Hospital for Sick Children, Great Ormond Street, and the Institute of Child Health, \\ Guilford Street, London
}

SUMMARY Two examples are presented of non-traumatic lesions of the deep motor branch of the ulnar nerve occurring in children. The clinical and histological features of solitary neurofibroma and neurilemmoma are compared and contrasted.

Soon after entry into the hand, the ulnar nerve divides into a superficial sensory branch and a deep motor branch; the latter supplies the hypothenar eminence, the interossei, the medial two lumbrical muscles, adductor pollicis and part of flexor pollicis brevis.

A lesion of the ulnar nerve in the hand may present clinically in one of five ways, depending upon the site of the lesion (Uriburu et al., 1976). If the lesion is proximal to the division into motor and sensory branches, there is weakness of all muscles supplied by the nerve and sensory loss (Brooks, 1952; Richmond, 1963). Brooks (1952) has also described a mixed motor and sensory deficiency syndrome sparing the hypothenar muscles. A lesion of the motor branch just distal to the division spares the sensory component but there is weakness of all muscles supplied by the nerve (Hunt, 1908). A lesion of the sensory branch alone gives rise to a pure sensory deficit (Shea and McClain, 1969). Finally a distal lesion of the motor branch may give rise to no sensory loss but weakness in all muscle groups except the hypothenar mass (Seddon, 1952).

Ramsay Hunt (1908) first described compressive lesions of the deep motor branch of the ulnar nerve and attributed them to neuritis secondary to occupational trauma. In 1952 Seddon reported four patients with compression of the nerve due to small ganglia emerging from the palmar aspect of a carpal joint. In the same year, Aubrey Jenkins reported three cases of solitary tumours arising from peripheral nerve trunks; one of these involved the deep branch of the ulnar nerve and was regarded as a neurilemmoma.

The purpose of this paper is to present two

Address for reprint requests: Dr N. Cavanagh, The Hospital for Sick Children, Great Ormond Street, London WC1N 3JH, England.

Accepted 14 March 1977 examples of non-traumatic lesions of the deep motor branch of the ulnar nerve oscurring in children. We can find no previous reference to such lesions in children and it is the clinical impression of D. Brooks, who cannot recall any in his own experience, that they must be exceedingly rare (personal communication). Our patients are also of interest in that each had a benign tumour of different type, and illustrate the contrasting clinical and histological features of solitary neurofibromas and neurilemmomas.

\section{Case 1}

(Hospital no. 426464)

A seven year old girl presented with a four month history of being unable to adduct the fingers of her right hand. This was first noticed in the swimming pool. During the four months the weakness had become more noticeable. There was no history of trauma, recent illness or pain, and she was still able to write normally at school.

In the past she had had no significant illness except for a spontaneously resolving cavernous haemangioma on the tip of the nose.

Family history was unremarkable and excluded von Recklinghausen's disease.

On examination she was normal except for the right hand. There was moderate wasting of the 1st dorsal interosseous muscle. The hypothenar eminence was normal. There was no fasciculation. There was weakness of abduction of the forefinger and weakness of adduction of the 1st, 2nd, 3rd, and 4th fingers. Power was normal in abductor pollicis brevis, finger flexors and extensors, and abductor digiti minimi. There was no loss of sensation in any modality and two point discrimination was normal. There was no palpable swelling. 
INVESTIGATIONS

The following were normal: full blood picture, radiographs of right hand, wrist and elbow, cervical spine, and chest. There was no radiological evidence of a cervical rib.

The first dorsal interosseous muscle was sampled for electromyography with a concentric needle electrode: no fibrillation was seen and no motor unit potentials were observed on volition or nerve stimulation. With surface electrodes at the thenar and hypothenar eminences, motor conduction velocity in the median and ulnar nerves was measured as normal. Median and ulnar sensory nerve action potentials were also normal ( $\mathrm{Dr} R$. Willison and NPCC).

At operation (Mr G. C. Lloyd-Roberts) the ulnar nerve was exposed above the wrist and traced down to the ulnar aspect of the hook of the hamate where it divided into three. There was the usual superficial branch which was normal but there were two deep branches. One was an ulnar branch going to part of the hypothenar eminence and the other a deep branch going anteriorly and across the palm with the deep palmar arch. This was a very large branch and the other was smaller than the usual deep branch. At the distal end of the branch going to the radial side, there was a definite swelling within the nerve. This swelling was hard, and on splitting the nerve it had the appearance of a neuroma. Three small specimens were taken for histology, fixed in neutral buffered formalin and embedded in paraffin wax. Sections cut at $5 \mu \mathrm{m}$ were stained with haematoxylin and eosin, haematoxylin-van Gieson mixture, Palmgren's method for nerve fibres, and solochrome cyanin for myelin.

Examination of the sections revealed an unencapsulated tumour consisting of plump fibroblasts, collagen, and axons, randomly distributed. Whorled structures suggestive of Schwann cell derivation were seen (Fig. 1). The presence of myelinated nerve fibres was confirmed by solochrome cyanin staining (Fig. 2). No nuclear pleomorphism, hyperchromatic nuclei, or mitoses were seen. The diagnosis was made of neurofibroma with no evidence of malignancy.

The child made an uneventful postoperative recovery and the wound healed well.

At follow-up four and nine months later, there had been some return of power of forefinger abduction. On EMG sampling at the site of the 1st dorsal interosseous muscle there appeared to be a few motor units under voluntary control.

\section{Case 2}

(Hospital no. 342675)

At 10 months of age a swelling appeared on this

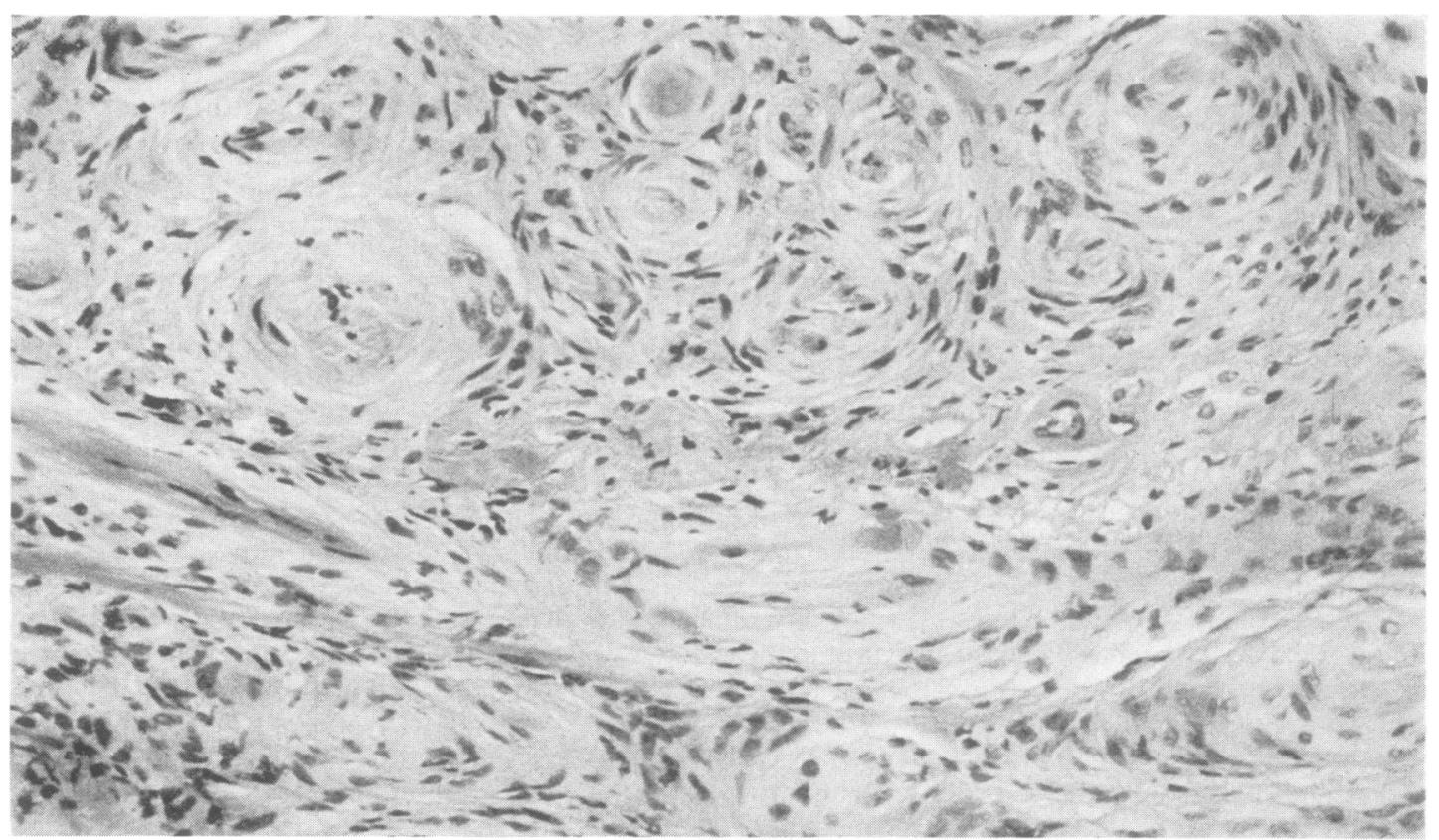

Fig. 1 Case 1. Haematoxylin and eosin $\times 300$. A neurofibroma containing fibroblasts, collagen, and whorled structures. 


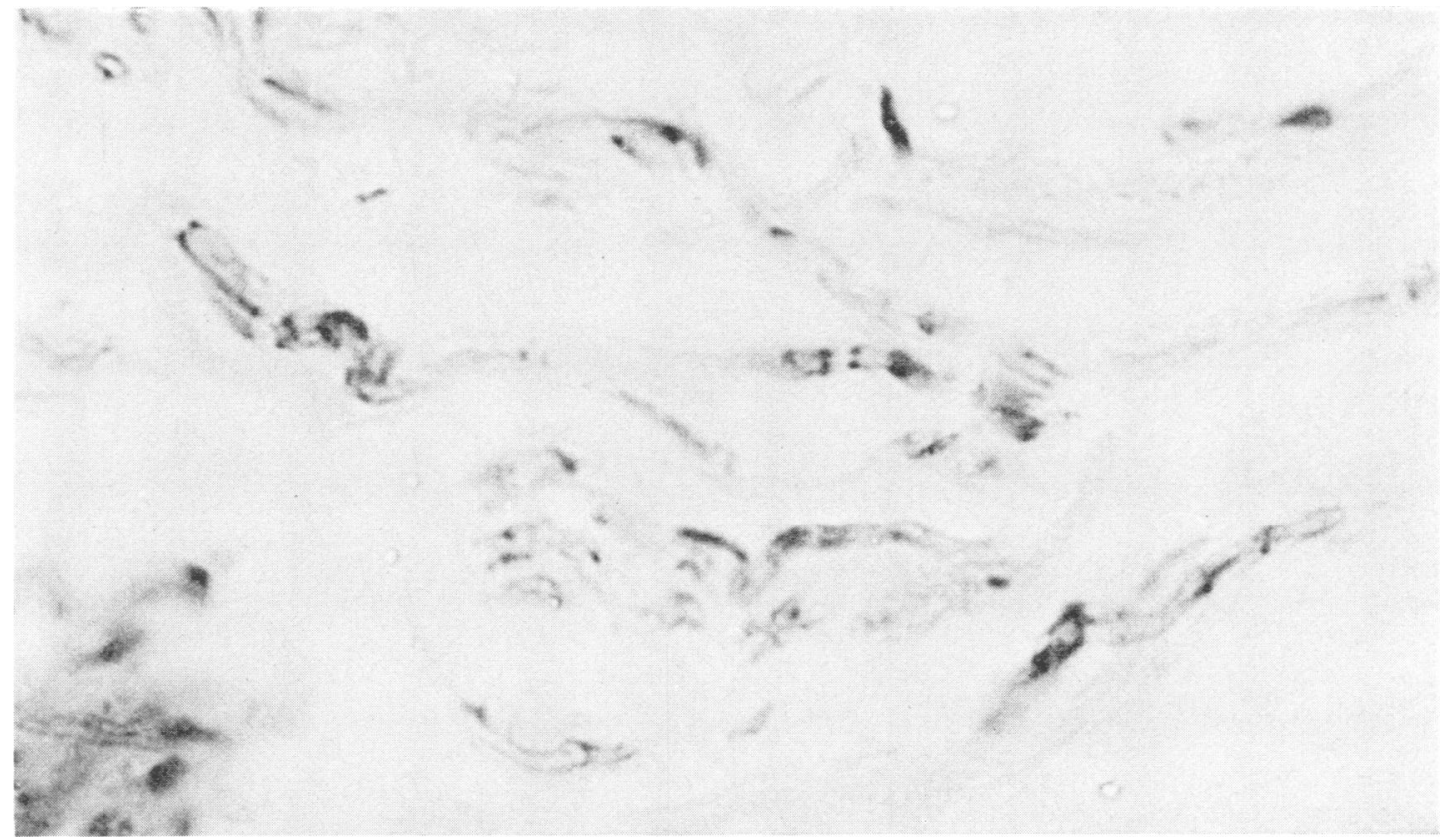

Fig. 2 Case 1. Solochrome cyanin $\times 960$. Myelinated nerve fibres are present.

girl's right hypothenar eminence. There was no previous history of trauma. It increased in size and was excised four months later. It was reported to be a neurofibroma. The exact site of the lesion and the extent of its removal is not known.

Eight months later the tumour recurred, and six months later it was re-explored. By then it was a mass $40 \mathrm{~mm}$ in diameter and was found to arise from the deep branch of the ulnar nerve. The sections were reviewed at this hospital, when the tumour was found to be a neurilemmoma, of nodular appearance (Fig. 3). It consisted entirely of a dense population of plump cells which demonstrated a nuclear pallisading, constituting Antoni A type tissue. No loose mucinous tissue of Antoni B type was seen (Fig. 4). The tumour extended to the margins of excision. Clinically the removal was thought to be incomplete. There was no postoperative ulnar paresis.

Eleven months after the second exploration and excision, the swelling recurred. The child was now $3 \frac{1}{2}$ years of age and was seen at this hospital for the first time. Examination of the hand revealed three small masses overlying the ulnar nerve. There was no muscle wasting and no sensory loss. Movements of the fingers were full. Full general and neurological examination revealed no abnormalities.

At operation (Mr D. N. Matthews) the lesions were excised and the ulnar nerve was sacrificed from just above the flexor retinaculum to a point $10 \mathrm{~mm}$ distal to the hook of the hamate where it divided into one deep, and two superficial branches. A split skin graft was applied. The tumour was fixed in neutral buffered formalin and embedded in paraffin wax. Histological sections were cut at $5 \mu \mathrm{m}$ and stained with haematoxylin and eosin, haematoxylin-van Gieson mixture, Palmgren's stain, and solochrome cyanin. Examination of the sections revealed a nodular neurilemmoma, consisting entirely of Antoni A tissue and similar in appearance to that seen in the first recurrence. There was a minimal histological margin of clearance, with tumour nodules in the overlying subcutaneous tissue and in the perineureum of the ulnar nerve. No nerve fibres were seen within the tumour, and there was no evidence of malignancy.

At the age of 4 years and 9 months, the wound was re-opened to explore a mass which had reappeared. Again, histological sections revealed a nodular neurilemmoma consisting of Antoni A tissue.

Two months later a mass appeared in the palm, distal to the previous operation site. At operation it was found to arise from the ulnar nerve. The tumour was removed together with the part of the ulnar nerve remaining at the distal end of the 


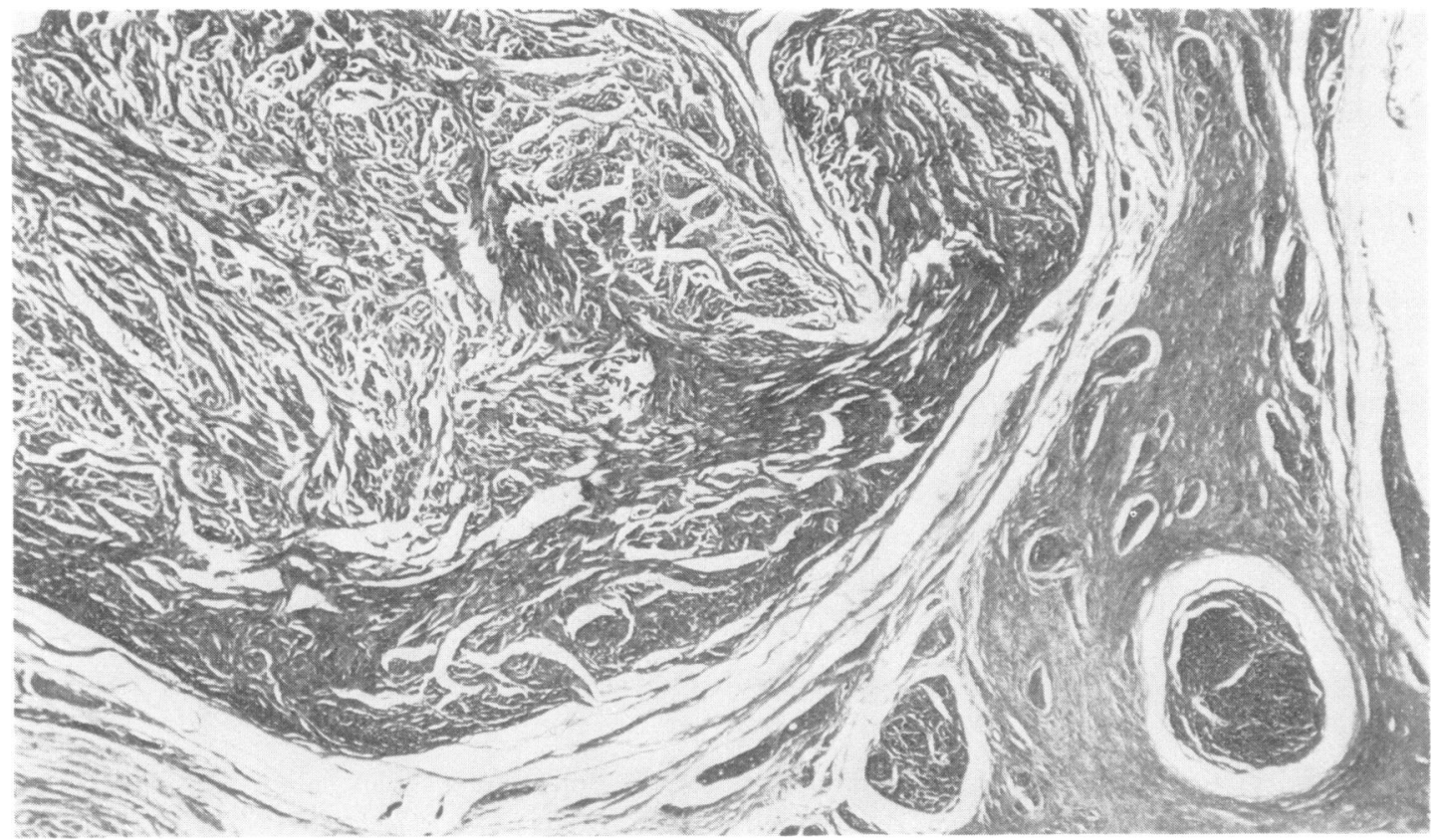

Fig. 3 Case 2. Haematoxylin and eosin $\times 20$. A neurilemmoma consisting of large and small nodules.

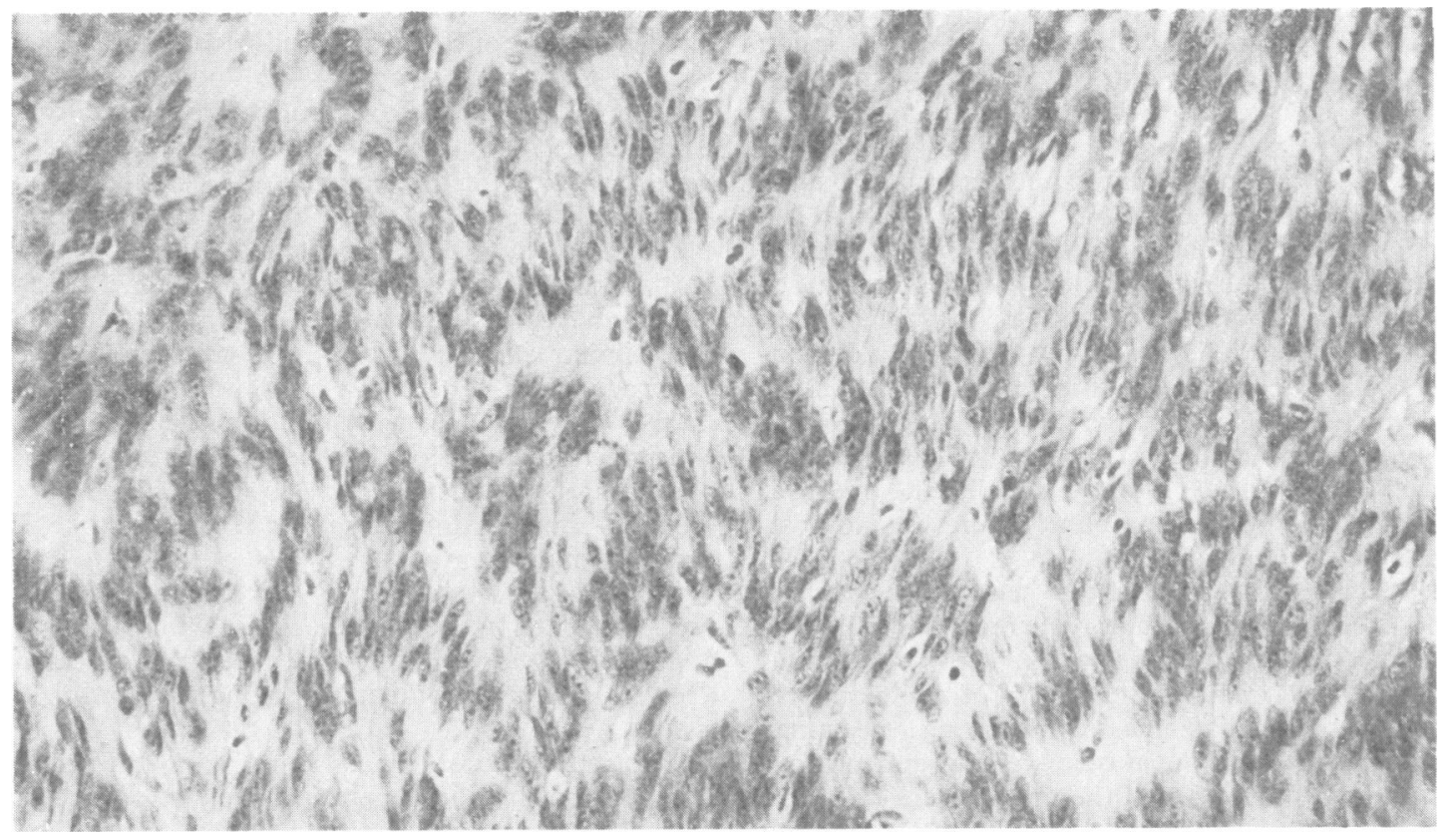

Fig. 4 Case 2. Haematoxylin and eosin $\times 300$. The cells of the neurilemmoma demonstrate pallisiding of plump nuclei constituting Antoni A tissue only. 
palm. Histology of the resected tumour revealed a nodular neurilemmoma extending to the margin of resection.

One year later, when aged 6 years, as no recurrence had been seen, the patient received a sural nerve graft. Subsequent examination revealed increasing recovery of muscle power and sensation in the ulnar nerve distribution.

At the age of 8 years she was admitted for excision of two small superficial swellings at the edge of the graft scar on the right hypothenar eminence. Histological examination of these revealed nodules of neurilemmoma.

When last seen three years ago (aged 14 years) there had been no clinical recurrence of tumour. The tip of the pulp of the fifth digit had no sensation and there was slight weakness of adduction of the fourth and fifth digits.

The above clinical details of this child were obtained from her notes. She has never been seen by the authors of this paper, though the histology of all the resections has been reviewed for the present comparison. She now lives abroad at an unknown address.

\section{Discussion}

The neurilemmoma and neurofibroma are the only true benign tumours to arise from nerve trunks.

Classically, the neurilemmoma is solitary, slow growing, and associated with radiating pain. There is a low incidence in childhood. Recurrences are rare (5\% in Heard's 1962 series). Although any association with von Recklinghausen's disease is thought to be rare, Stout (1935) found three definite and six probable cases in a series of 50 . Neurilemmomas are said to occur eccentrically on their nerve of origin, causing lateral displacement of nerve fibres. Though no nerve fibres were seen in our case the significance of this is difficult to assess, as material from the original resection was not available for examination.

By contrast, the neurofibroma, though sometimes solitary, is more often part of von Recklinghausen's disease. In Seddon's cases (1972) the hand was a common site for solitary presentation. Most are superficial and present as a swelling. There is a high incidence in childhood. Recurrence is rare when excision is complete. While malignant transformation may occur in the larger or deeper neurofibromas the small superficial tumours are seldom susceptible to this. Neurofibromas are said to arise within the nerve trunk of origin and to spread by infiltration. The tumour will, therefore. often contain nerve fibres as was seen in our case 1 . Neither of the children presented here had any associated features of von Recklinghausen's disease, though in case 1 there was a history of a spontaneously resolving cavernous haemangioma of the nose.

The presentation of the neurilemmoma might be considered atypical, in that pain was never a feature. There is, however, enough evidence in the literature to suggest that pain is not invariable. (Stout, 1935; Saxen, 1948; Heard, 1962). In retrospect it is likely that the repeated recurrence in our case 2 was the result of inadequate excision due to the nodular nature of the tumour (Fig. 3).

The position of the neurofibroma supports Seddon's (1972) observation that the hand is a relatively common site for solitary peripheral nerve tumours. The presentation was atypical in that there was no swelling, but this may simply be the result of early diagnosis.

Finally, it is worth emphasising that in case 1 we were unable to find any active muscle fibres in the 1st dorsal interosseous muscle, in contrast to the normal studies in the hypothenar eminence. This strongly favoured a lesion of the deep motor branch of the ulnar nerve. However, it is more common to find reduced conduction velocity in the ulnar nerve to the 1st dorsal interosseous, compared with a normal velocity to the hypothenar eminence (Simpson, 1956). The electrical silence of the 1st dorsal interosseous in our case 1 may indicate that the tumour had been present since earty life.

We would like to thank Dr E. Brett and Mr D. N. Matthews for allowing us to report these patients, Dr R. Willison for advice with electromyography, and $\mathrm{Mr}$ G. C. Lloyd-Roberts for his great help in the preparation of this paper.

\section{References}

Brooks, D. M. (1952). Nerve compression by simple ganglia. Journal of Bone and Joint Surgery, 34B, 391-400.

Heard, G. (1962). Nerve sheath tumours and von Recklinghausen's disease of the nervous system. Annals of the Royal College of Surgeons, 31, 229248.

Hunt, J. R. (1908). Occupation neuritis of the deep branch of the ulnar nerve. A well-defined clinical type of profession palsy of the hand. Journal of Nervous and Mental Diseases, 35, 673-689.

Jenkins, S. A. (1952). Solitary tumours of peripheral nerve trunks. Journal of Bone and Joint Surgery, 34B, 401-411.

Richmond, D. A. (1963). Carpal ganglion with ulnar nerve compression. Journal of Bone and Joint Surgery, 45B, 513-515. 
Saxen, E. (1948). Tumours of the sheaths of the peripheral nerves (studies on their structure, histogenesis and symptomatology). Acta Pathologica et Microbiologica Scandinavica, Supplement 79.

Seddon, H. J. (1952). Carpal ganglion as a cause of paralysis of the deep branch of the ulnar nerve. Journal of Bone and Joint Surgery, 34B, 386-390.

Seddon, Sir Herbert (1972). Surgical Disorders of the Peripheral Nerves, page 164. Churchill Livingstone: Edinburgh.

Shea, J. D., and McClain, E. J. (1969). Ulnar nerve compression syndrome at and below the wrist. Journal of Bone and Joint Surgery, 51A, 1095-1103.
Simpson, J. A. (1956). Electrical signs in the diagnosis of carpal tunnel and related syndrcmes. Journal of Neurology, Neurosurgery, and Psychiatry, 19, 275280.

Stout, A. P. (1935). The peripheral manifestations of the specific nerve sheath tumor (neurilemmoma). American Journal of Cancer, 24, 751.

Uriburu, I. J. F., Morchio, F. J., and Marin, J. C. (1976). Compression syndrome of the deep motor branch of the ulnar nerve (piso-hamate hiatus syndrome). Journal of Bone and Joint Surgery, 58A, 145-147. 